\title{
Footprints of deformation mechanisms during in situ x-ray diffraction: Nanocrystalline and ultrafine grained $\mathbf{N i}$
}

\author{
Z. Budrovic, S. Van Petegem, P. M. Derlet, B. Schmitt, and H. Van Swygenhoven ${ }^{\text {a) }}$ \\ Paul Scherrer Institute, CH-5232 Villigen-PSI, Switzerland \\ E. Schafler and M. Zehetbauer \\ Materials Physics Institute, University of Vienna, A-1090 Wien, Austria
}

(Received 10 January 2005; accepted 6 May 2005; published online 3 June 2005)

\begin{abstract}
In situ $\mathrm{x}$-ray diffraction demonstrates that in ultrafine grained $\mathrm{Ni}$ synthesized by high pressure torsion with a coherent scattering domain size of $80 \mathrm{~nm}$, a dislocation network is still built up during tensile deformation whereas this is not the case for electrodeposited nanocrystalline metals with a coherent scattering domain size of $30 \mathrm{~nm}$. Simultaneously, the technique shows for the first time important differences in macroscopic stress accommodation during plastic deformation between the nanocrystalline and ultrafine grained $\mathrm{Ni}$, such as the origin of the reduction in flow stress. (C) 2005 American Institute of Physics. [DOI: 10.1063/1.1947385]
\end{abstract}

There is a long-standing discussion whether plasticity is still governed by dislocation mechanisms in nanocrystalline (nc) metals, ${ }^{1,2}$ where the conventional dislocation theory predicts the existence of a critical grain size below which a dislocation source can no longer operate. ${ }^{3}$ At these small grain sizes, the small amount or total absence of work hardening is usually explained by the lack of dislocation generation and multiplication mechanisms, resulting in a reduction of flow stress and the early onset of necking. Several postmortem techniques have been applied to show dislocation activity, ranging from conventional x-ray diffraction (XRD) analysis to in situ transmission electron microscopy (TEM) studies. ${ }^{4,5}$ It is well known that the presence of dislocations contributes to peak broadening in XRD patterns, and elaborate physical models have been applied to calculate the dislocation density from XRD peak widths. ${ }^{6}$ These types of analyses result in a very high dislocation density prior to deformation since the technique cannot distinguish between the grain boundary and lattice dislocations. Recently, we have demonstrated a reversible peak broadening in electrodeposited (ED) Ni during in situ peak profile analysis at the Swiss Light Source (Paul Scherrer Institut, Villigen, Switzerland), a technique that follows continuously peak position and peak broadening [represented by the full width half maximum (FWHM)] during mechanical deformation. ${ }^{7}$ In Ref. 7, the sample was sequentially loaded and unloaded in the beam with the profile of several diffraction peaks being measured continuously during the loading and unloading cycles. The observation that the peak broadening is reversible upon unloading demonstrated that no permanent residual dislocation network can be built up during plastic deformation in the $26 \mathrm{~nm}$ grain size ED Ni sample.

In this letter, we report on the different in situ XRD peak profile behavior of nc ED Ni and ultrafine grained high pressure torsion (HPT) Ni during elastic and plastic loading, revealing their fundamental differences in terms of strain accommodation and plastic deformation mechanisms.

The HPT Ni is $99.9 \%$ pure, and the material was synthesized in the form of disks of $8 \mathrm{~mm}$ diameter and a thickness of $0.8 \mathrm{~mm}$ using a pressure of $8 \mathrm{GPa}$ with two rotations lead-

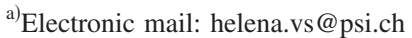

ing to a van Mises strain of approximately 36 in peripheral sample areas. The microstructure obtained using HPT synthesis methods ${ }^{8}$ is very different from that of ED Ni. TEM analysis performed by us showed large grains (200-400 nm) with a visible subgrain boundary structure resulting in a maximum coherent XRD domain size of the order of $80 \mathrm{~nm} .{ }^{9}$ The microstructure of the ED Ni is characterized by a narrow grain size distribution made from equiaxed grains. ${ }^{7}$ Here, a mean grain size of $26 \mathrm{~nm}$ was obtained from a WilliamsonHall analysis of the XRD pattern, and corresponds well with the grain sizes observed in TEM analysis. Cross-sectional analysis did not show columnar growth.

For HPT Ni, we have performed a loading/unloading experiment (see Fig. 1) similar to that reported in Ref. 7 for ED Ni. Only the results for the (222) diffraction peak are shown, the behavior for the other peaks being very similar. A maximum flow stress of $1.4 \mathrm{GPa}$ is reached (compared to 2.2 GPa in ED Ni). During the last loading cycle, the flow stress decreases linearly toward a value of $1 \mathrm{GPa}$, where fracture occurs at a total strain of $8.5 \%$. Observation by a digital camera mounted close to the sample stage revealed the formation of a neck during the last loading cycle. The neck became visible at about $4 \%$ total strain. This is in contrast to the observations for $\mathrm{ED} \mathrm{Ni}$, where no pronounced neck could be observed within the resolution of the camera, although the flow stress also decreased prior to failure. During all loading curves for both HPT Ni and ED Ni [shown in the inset of Fig. 1(d)], the specimens were plastically deformed.

In contrast to ED Ni, the peak broadening of HPT Ni is clearly not fully reversible. During the first loading cycle, the FWHM increases by 55\% recovering to $30 \%$ upon unloading, and during the second loading, the FWHM increases by $70 \%$ recovering to $40 \%$. In the third loading cycle, the maximum FWHM is reached (74\%) with a recovery until $45 \%$ (all percentages are related to the initial value of FWHM $=0.1$ ). During the last loading cycle, the FWHM rises again to its maximum value and stays approximately constant during further straining until $8.5 \%$. The irreversible FWHM can be assigned to the formation of dislocation structures during homogenous deformation, since irreversibility is observed well before the maximum flow stress is reached and before 

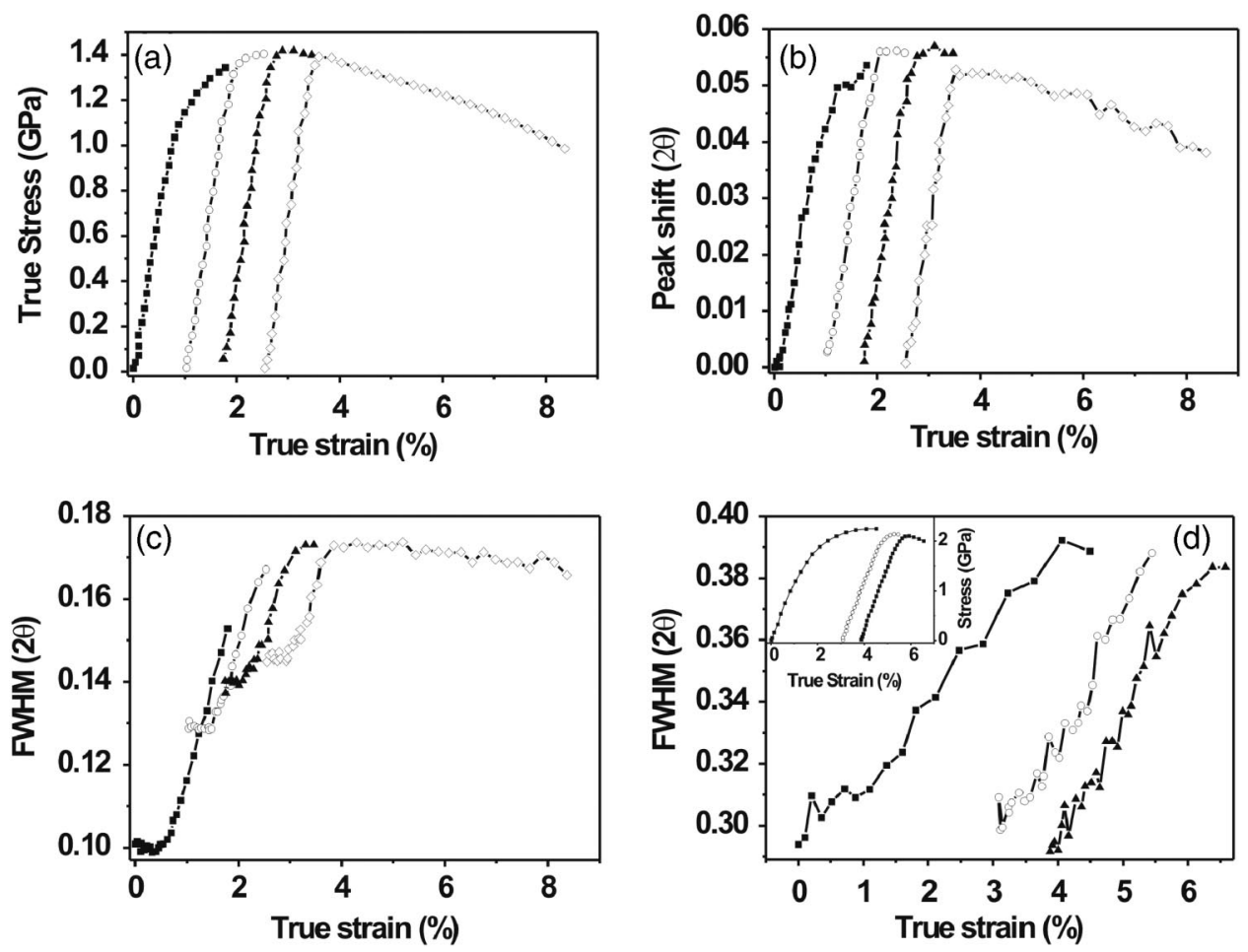

FIG. 1. (a) The stress-strain curve of HPT Ni, (b) the peak shift of HPT Ni and the FWHM of the (222) diffraction peak for (c) HPT Ni and (d) ED Ni. neck formation. TEM investigations after deformation do not reveal major changes in grain or subgrain distributions. This is to be expected since the increase in dislocation density that can be associated with the irreversible part of the FWHM during a total strain of $8 \%$ is relatively small compared to the initial dislocation density. ${ }^{10}$ Due to the local character of TEM observations, such small increases in dislocation densities can not be observed, demonstrating once more the power of the in situ technique.

The partial reversibility of the peak broadening shows clear evidence that there are two sources of inhomogeneous strains that contribute to the FWHM during plastic deformation of HPT Ni: The first coming from elastic inhomogeneous strains that are reversible upon unloading, the second from inhomogeneous strains arising from an additional permanent dislocation network that can still be built up during tensile deformation of the ultrafine grained HPT structure. It is important to note that during the actual straining to $8.5 \%$, the coherent scattering domain size varied only within the experimental error. ${ }^{9}$

Besides gaining new information on dislocation mechanisms, this type of in situ XRD peak profile analysis can also shed light on differences in stress accommodation during plastic deformation. Indeed, for both ED Ni and HPT Ni, the peak positions during loading/unloading cycles are found to be fully reversible, behaving approximately linear with increasing stress up to the maximum flow stress. Figure 2. displays the peak shift as a function of applied stress until failure for a selected number of Bragg peaks for [Fig. 2(a)] HPT Ni and [Fig. 2(b)] ED Ni, where the arrows pointing to the right indicate loading until the respective maximum flow stresses. Figure 2 displays a range of applied stresses chosen to better display the maximum flow stress region. After the maximum flow stress has been reached, the peak shift in HPT Ni decreases linearly with the flow stress (reducing by $500 \mathrm{MPa}$ before failure), whereas in ED Ni the peak shift remains approximately constant with respect to the decreasing flow stress (reducing by $300 \mathrm{MPa}$ before failure). This part of the loading is represented by the arrows now pointing to the left in Fig. 2. In the case of HPT Ni, when considering all observed peaks, the ratio of the gradients before and after maximum flow stress are different for the different families of reflection. In the case of $\mathrm{ED} \mathrm{Ni}$, the constant peak shift after maximum flow stress suggests that the reduction in flow stress observed in ED-Ni is not carried by the grain interior (the coherent scattering volume) but is a result of stress accommodation in the grain boundary network. This could be, for instance, related to the formation of nanovoids, ${ }^{11}$ or some other damage, that would reduce the effective cross section without visible neck formation. TEM investigation of the
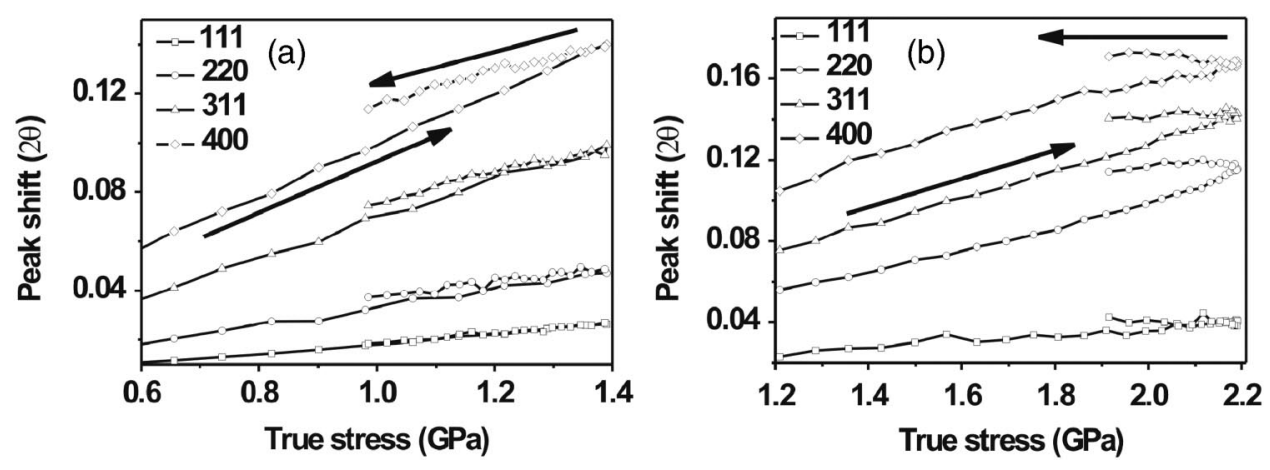

FIG. 2. Peak shift of the (111), (220), (311), and (400) peak as function of stress for (a) HPT Ni (last loading cycle of Fig. 1) and (b) ED Ni (from a continuous loading experiment). The arrows pointing to the right indicate loading until maximum flow stress, and the arrows pointing to the left indicate loading beyond maximum flow stress. 
postdeformed sample did not however reveal any notable difference in microstructure. ${ }^{7}$ These observations indicate that the softening that is observed in both materials-after the maximum flow stress has been reached-is of a different origin and this is also reflected by the absence of a visible neck in ED Ni.

The in situ observations revealed important differences in elastic and plastic deformation behavior in nc nd ultrafine grained metals. The results clearly demonstrate that in an ultrafined grained HPT structure dislocation networks can still be built up during tensile deformation, whereas in nc structures with a mean grain size of $30 \mathrm{~nm}$ this is no longer possible. Moreover, the presented technique allows the gathering of unique information on strain accommodation during elastic and plastic deformation not accessible by other postmortem experimental techniques
${ }^{1}$ J. R. Weertman, Mechanical Behaviour of Nanocrystalline Metals in Nanostructured Materials: Processing, Properties, and Potential Applications (William Andrew Publishing, Norwich, CT, 2002).

${ }^{2}$ K. S. Kumar, H. Van Swygenhoven, and S. Suresh, Acta Mater. 51, 5743 (2003).

${ }^{3}$ M. Legros, B. R. Elliott, M. N. Rittner, J. R. Weertman, and K. J. Hemker, Philos. Mag. A 80, 1017 (2000).

${ }^{4}$ C. Hugo, H. Kung, J. R. Weertman, R. Mitra, J. A. Knapp, and D. M. Follstaedt, Acta Mater. 51, 1937 (2003).

${ }^{5}$ K. S. Kumar, S. Suresh, M. F. Chisholm, J. A. Horton, and P. Wang, Acta Mater. 51, 387 (2003).

${ }^{6}$ T. Ungar, A. Revesz, and A. Borbely, J. Appl. Crystallogr. 31, 554 (1998).

${ }^{7}$ Z. Budrovic, H. Van Swygenhoven, P. M. Derlet, S. Van Petegem, and B. Schmitt, Science 304, 273 (2004).

${ }^{8}$ R. Z. Valiev, R. K. Islamgaliev, and I. V. Alexandrov, Prog. Mater. Sci. 45, 103 (2000).

${ }^{9}$ E. Schafler and R. Pippan, Mater. Sci. Eng., A 387, 799 (2004).

${ }^{10}$ E. Schafler, M. Zehetbauer, and T. Ungar, Mater. Sci. Eng., A 319, 220 (2001).

${ }^{11}$ K. S. Kumar, S. Suresh, M. F. Chrisholm, J. A. Horton, and P. Wang, Acta Mater. 51, 387 (2003). 